\title{
Influencia del proceso analítico en la aparición y desaparición de los espermatozoides del semen tras la vasectomía
}

\author{
Cháfer Rudilla M, Navarro Casado L, Belilty Araque M, Andrés Fernández C, Quintanilla Mata M. \\ Laboratorio de Análisis Clínicos. Complejo Hospitalario y Universitario de Albacete.
}

Actas Urol Esp. 2007;31(3):270-275

\begin{abstract}
RESUMEN
INFLUENCIA DEL PROCESO ANALÍTICO EN LA APARICIÓN Y DESAPARICIÓN DE LOS ESPERMATOZOIDES DEL SEMEN TRAS LA VASECTOMÍA

Objetivo: Evaluar si el proceso analítico puede justificar que en algunos pacientes se observen espermatozoides residuales en algunos de sus espermiogramas para el control de la vasectomía, pero no en todos.

Material y métodos: Se revisaron los controles de las vasectomías realizadas desde enero de 2002 a diciembre de 2004. Nuestro protocolo consiste en una adaptación a nuestro medio de las recomendaciones de la Sociedad Británica de Andrología para el control de la vasectomía y de la Organización Mundial de la Salud para el análisis seminal.

Resultados: Durante los 3 años del estudio se ha intervenido a 984 pacientes de vasectomía y se recibieron una media de 2 muestras por paciente. En relación a la calidad de la fase preanalítica, 134 pacientes $(9,4 \%)$ refirieron no haber recogido todo el eyaculado; los volúmenes de semen fueron inferiores a $2.0 \mathrm{~mL}$ en 269 especímenes $(18,8 \%$ ) y existían dudas de que los eyaculados fueran completos; en 11 casos, las condiciones preanalíticas fueron incorrectas: contenedores mal etiquetados, derramados o inadecuados. Con respecto a la fase analítica, en 432 eyaculados $(30,5 \%)$ se encontraron alteraciones de la consistencia; 62 especímenes $(4,3 \%)$ contenían abundantes células y obstruían la visualización microscópica. En cuanto a la fase postanalítica, 153 pacientes $(20,9 \%)$ obtuvieron informes con resultados alternativos de azoospermia y de presencia de espermatozoides residuales.

Conclusiones: Se produce un elevado porcentaje de incidencias en la fase preanalítica y la analítica en los controles de los pacientes vasectomizados. Estas condiciones inadecuadas pueden afectar los resultados y justificar que los espermatozoides residuales se observen o no en los eyaculados de algunos pacientes. Recomendamos realizar un mínimo de dos espermiogramas por paciente para asegurar su correcta evaluación. Proponemos informar los resultados negativos como un recuento inferior al límite de detección de nuestra técnica de manera similar a otras magnitudes del laboratorio para minimizar el efecto de la fluctuación de los recuentos en estos niveles tan bajos.

Palabras clave: Control de vasectomía. Proceso analítico. Espermatozoides residuales.
\end{abstract}

\section{ABSTRACT}

INFLUENCE OF THE ANALYTICAL PROCESS IN THE APPEARANCE AND DISAPPEARANCE OF THE SPERMATOZOA AFTER VASECTOMY

Objectives: To evaluate if the analytical process might justify that in some patients rare non motile sperm might be seen in some but not all their post-vasectomy semen samples.

Patients and methods: Post vasectomy ejaculates received in our Center from january 2002 to december 2004 were reviewed. We used our own guidelines for post vasectomy semen assessment based upon those of the British Andrology Society for the evaluation of post vasectomy semen samples and the World Health Organization guidelines for semen analysis.

Results: During the 3 years of follow up, 984 patients underwent vasectomy. We received 1.430 semen samples, 2 samples per patient on average. Regarding the pre analytical phase, 134 samples (9.4\%) were not completely collected; ejaculate volumes of less than $2 \mathrm{~mL}$ were delivered by 269 patients (18.8\%); in these cases, we were not sure whether the whole ejaculates were submitted; pre analytical conditions of 11 samples $(0.8 \%)$ were inappropriate: incorrectly labeled, spilled, provided into inadequate containers... Regarding the analytical phase, 432 ejaculates $(30.5 \%)$ were extremely viscous and sperm detection might have been affected; 62 semen samples (4.3\%) contained many cells which obstructed the visualization of the entire microscopy field. Regarding the post analytical phase, 153 patients $(20.9 \%)$ had alternative negative/positive results with rare non motile sperm.

Conclusion: An elevated percentage of incidences involving both the pre analytical and the analytical phase were observed during post-vasectomy seminal analysis. Inadequate conditions may affect the results and justify that spermatozoa may be seen in some but not all the ejaculates of the same patient. We recommend that two semen samples per patient are required to ensure that he is correctly evaluated. We propose to report a negative result as a spermatozoa count bellow the detection limit of our analytical procedure similar to other laboratory magnitudes to minimize the effect of fluctuations in such a low count of rare non motile sperm.

Keywords: Vasectomy assessment. Analytical process. Rare non motile sperm. 
$\mathrm{A}$ ctualmente no hay duda de que el análisis del semen tras la intervención de vasectomía es absolutamente imprescindible como herramienta para asegurar el éxito de la cirugía y es indiscutiblemente útil como garantía legal para los urólogos que la realizan. Por un lado, los fallos inherentes a la intervención, la existencia de espermatozoides residuales y las recanalizaciones pueden conducir a embarazos no deseados que podrían haberse evitado con un sencillo espermiograma. En otras ocasiones, a pesar de haber conseguido un resultado negativo, en caso de producirse una gestación, el espermiograma nos sirve de prueba legal de que se habían seguido las recomendaciones en cuanto a la comprobación del éxito de la intervención ${ }^{1}$.

También es ampliamente conocido que en un porcentaje elevado de pacientes vasectomizados no se alcanza la azoospermia, sino que se siguen observando espermatozoides inmóviles residuales en sus eyaculados ${ }^{2}$. No está claro cuál es el origen de dichos espermatozoides, aunque se cree que pasan a través de pequeños canales y hendiduras originados en el tejido conectivo y cicatrizal localizado entre los dos extremos de los conductos deferentes seccionados. Lo que sí es indiscutible es que son objeto de controversia, han complicado considerablemente la consecución del alta deseable de estos pacientes e, incluso, han sido la causa de numerosas reintervenciones. Además, esta pequeña cantidad de espermatozoides inmóviles puede observarse incluso en pacientes previamente dados de alta con un resultado de azoospermia y, por este motivo, se pueden encontrar en la literatura expresiones tales como "reaparición de espermatozoides" tras la intervención de vasectomía ${ }^{2,3}$.

Por otro lado y, a pesar de los avances en la estandarización que se han producido en los últimos años, todavía existen grandes diferencias en las técnicas de laboratorio encaminadas a comprobar la eliminación de los espermatozoides de los conductos eyaculatorios. Por este motivo, nos planteamos como objetivo del presente estudio intentar dilucidar si la aparición y desaparición de espermatozoides del eyaculado en este grupo de pacientes sometidos a la intervención de vasectomía es real o puede obedecer, simplemente, a problemas relacionados con el proceso analítico.

\section{MATERIAL Y MÉTODOS}

Realizamos un estudio retrospectivo recogiendo los datos de todos los controles de vasectomía correspondientes a las intervenciones realizadas entre enero de 2002 y diciembre de 2004, descargándolos del Sistema de Información del Laboratorio Omega 2000 y se consultó con la documentalista del Hospital el número de vasectomías incluidas en dicho periodo.

Nuestro protocolo para el control de la vasectomía es una adaptación ${ }^{4}$ a nuestro medio de la guía de la Sociedad Británica de Andrología $(B A S)^{5}$, que incluye las recomendaciones de la Organización Mundial de la Salud sobre la recogida de semen para el estudio de fertilidad ${ }^{6}$. La hoja con las instrucciones para la recogida de semen se incluye en la Figura 1 y lleva, en la parte inferior de la misma, un cuestionario de calidad preanalítca para recoger datos que nos ayuden a realizar el informe de los resultados. El análisis seminal consiste en la visualización en un microscopio de contraste de fases de 10 $\mu \mathrm{L}$ de semen fresco, previamente homogeneizado, revisando la preparación completa (correspondiente a unos 300 campos, aproximadamente, a 200 aumentos, con ocular de $22 \mathrm{~mm}$ ). En aquellas muestras en las que se observan espermatozoides en casi todos los campos, se procede al recuento en la cámara de Neubauer, con o sin dilución previa, según la cantidad. Los eyaculados en los que no se observan espermatozoides en el análisis directo se centrifugan a más de 3.000 g durante 15 minutos, se decantan, se resuspende el sedimento y se toman otros $10 \mu \mathrm{L}$ para realizar un nuevo examen. A todos los sémenes que tienen espermatozoides inmóviles se les realiza una tinción de integridad de membrana con eosina para comprobar su vitalidad.

A los especimenes viscosos se les fuerza el paso a través de una pipeta Pasteur y, si esto no funciona, a través de una aguja de $0,8 \mathrm{~mm}$ con el fin de ayudar a licuar el semen y que la alícuota que observemos sea representativa, tratando de evitar que queden espermatozoides atrapados en el moco. En aquellos casos en los que no se vean espermatozoides, pero sí abundantes leucocitos, otro tipo de células o material amorfo (esmegma) que puedan dificultar la 


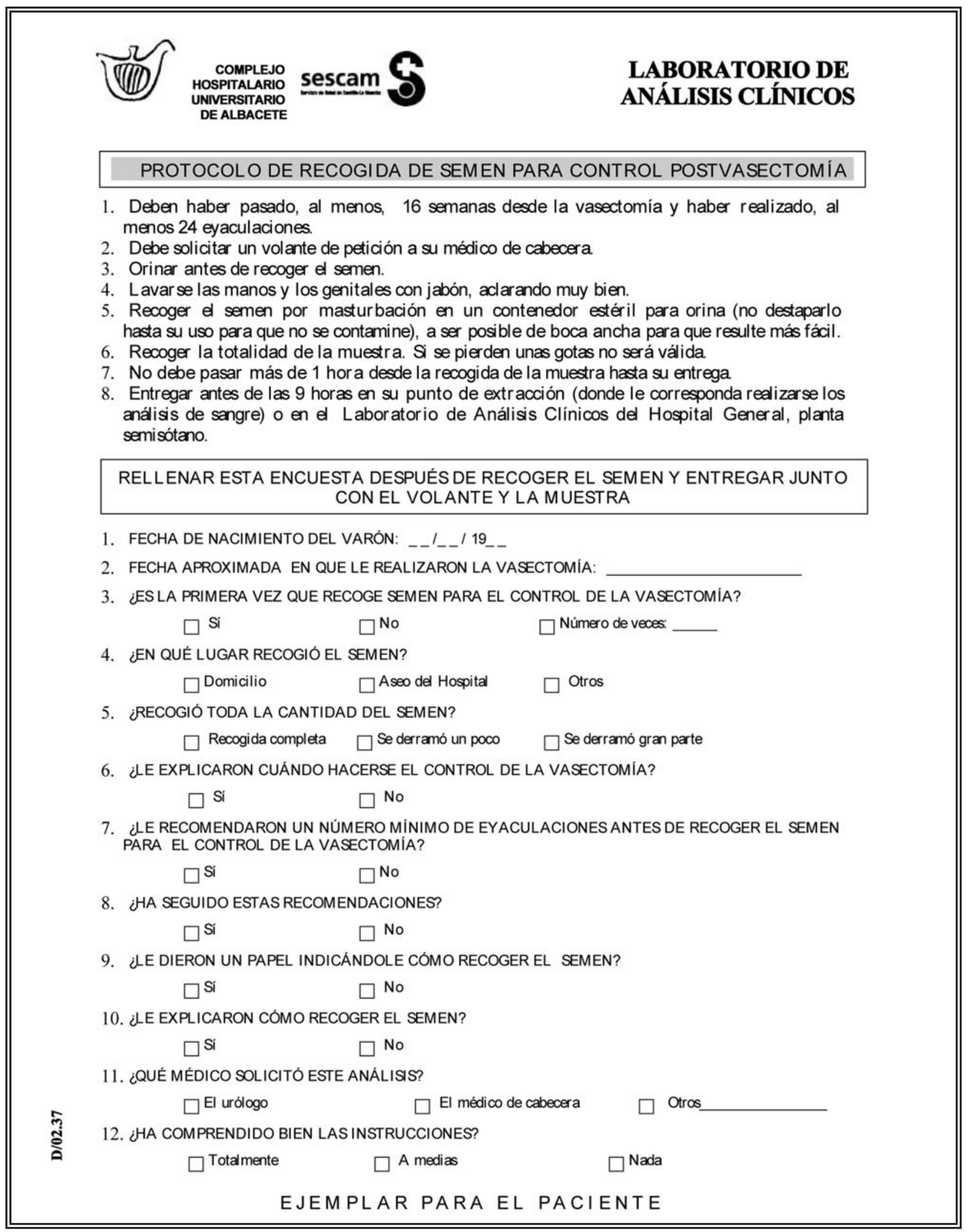

FIGURA 1. Instrucciones preanaliticas para recogida de semen para el control de la vasectomía del Complejo Hospitalario y Universitario de Albacete. 
visualización, se solicita la recogida de un nuevo eyaculado siguiendo estrictamente el protocolo preanalítico, con el fin de obtener una muestra lo más limpia posible.

De acuerdo con las recomendaciones de la BAS, a los pacientes que traen un espécimen en el cual no se observan espermatozoides, se les solicita un nuevo espermiograma en un mes y, si se trata del segundo semen sin espermatozoides, se les indica en el informe que no es necesario que traigan más eyaculados. A los pacientes que sí tienen espermatozoides, se les solicita un nuevo control al mes hasta que cumplan los criterios de alta aconsejados por la BAS. Del mismo modo, cuando ya han pasado más de 6 meses y se siguen observando espermatozoides móviles, se recomienda consultar con el Servicio de Urología. Sólo en aquellos casos en que, a pesar de seguirse observando espermatozoides vivos, ha disminuido drásticamente su número o ya no son móviles, se solicita un nuevo control, aunque hayan transcurrido más de 6 meses desde la intervención.

Las variables continuas se presentan como media \pm desviación estándar; las variables categóricas se expresan como número y porcentaje. Se empleó la prueba de Kolmogorov-Smirnov para comprobar la normalidad de las variables continuas.

\section{RESULTADOS}

Durante el periodo del estudio, en nuestro Hospital, se practicó la intervención de vasectomía a 984 pacientes; además, incluimos los datos de 67 pacientes que se realizaron los controles de vasectomía en nuestro laboratorio, pero fueron operados en otros centros. En total, tuvimos 731 pacientes, de los cuales se analizaron 1.430 muestras de semen, con una media de 2,0 muestras por paciente, y un tiempo medio de envío de la primera muestra a las 16 semanas (SD 9,3 semanas). Las incidencias que se presentaron en relación a la fase preanalítica se resumen en la Tabla $1 \mathrm{y}$, las que tuvieron lugar en la fase analítica, se reflejan en la Tabla 2. De los 732 pacientes, $153(20,9 \%)$ obtuvieron resultados alternativos de azoospermia y de presencia de espermatozoides muertos en concentraciones inferiores a 10.000 espermatozoides $/ \mathrm{mL}$.
Tabla 1

Incidencias preanaliticas

\begin{tabular}{|c|c|c|c|c|}
\hline \multicolumn{2}{|c|}{ Incidencia } & \multirow{2}{*}{$\begin{array}{c}\mathbf{N} \\
134\end{array}$} & \multirow{2}{*}{$\begin{array}{c}\% \\
32,4\end{array}$} & \multirow{2}{*}{$\begin{array}{c}\begin{array}{c}\% \text { del total } \\
\text { de eyaculados }\end{array} \\
9,4\end{array}$} \\
\hline $\begin{array}{l}\text { Muestra } \\
\text { incompleta }\end{array}$ & $\begin{array}{l}\text { Recogida } \\
\text { incompleta }\end{array}$ & & & \\
\hline & $\begin{array}{l}\text { Derramado } \\
\text { transporte }\end{array}$ & 5 & 1,2 & 0,3 \\
\hline Volumen & $\leq 2,0 \mathrm{y} \leq 1,0 \mathrm{~mL}$ & 149 & 36,0 & 18,8 \\
\hline inferior a & $\leq 1,0 \mathrm{y} \leq 0,5 \mathrm{~mL}$ & 30 & 7,2 & 2,1 \\
\hline $2,0 \mathrm{~mL}$ & $\leq 0,5 \mathrm{~mL}$ & 90 & 21,7 & 6,3 \\
\hline \multirow{3}{*}{$\begin{array}{l}\text { Contenedor } \\
\text { inadecuado }\end{array}$} & Tubo para orina & 3 & 0,7 & 0,2 \\
\hline & Preservativo & 1 & 0,2 & $<0,1$ \\
\hline & Papel de aluminio & 1 & 0,2 & $<0,1$ \\
\hline \multicolumn{2}{|c|}{ Sin identificar } & 1 & 0,2 & $<0,1$ \\
\hline \multicolumn{2}{|c|}{ Total } & 414 & 100 & 37,1 \\
\hline
\end{tabular}

Tabla 2

Incidencias analíticas

\begin{tabular}{llccc}
\hline \multicolumn{2}{c}{ Incidencia } & N & \% & $\begin{array}{c}\text { \% del total } \\
\text { de eyaculados }\end{array}$ \\
\hline $\begin{array}{llccc}\text { Dificultades } \\
\text { visualización de } \\
\text { espermatozoides }\end{array}$ & Leucocito/células & 62 & 12,5 & 4,3 \\
Alteraciones de la & Viscosidad elevada & 287 & 58,0 & 20,1 \\
consistencia & $\begin{array}{l}\text { Viscosidad muy } \\
\text { elevada }\end{array}$ & 135 & 27,3 & 9,4 \\
& $\begin{array}{l}\text { Licuefacción alterada } \\
\text { Total }\end{array}$ & 10 & 2,0 & 0,7 \\
& Tota & 100 & 33,5 \\
\hline
\end{tabular}

\section{DISCUSIÓN}

Parece bien establecido que la única manera de confirmar el éxito de la intervención de vasectomía es la realización de espermiogramas para comprobar que se ha conseguido la esterilidad. Sin embargo, a pesar de que en los últimos años han existido intentos de estandarización, el análisis de semen tras la intervención sigue siendo un campo del laboratorio en el que existen amplias variaciones, que repercuten de manera evidente tanto en los resultados como en su interpretación.

Es muy llamativo que, en nuestros cuestionarios de calidad preanalítica, un 9\% de los pacientes reconoce que se le ha derramado una pequeña parte de la muestra durante la recogida. Aún es más curioso comprobar que, según se observa 
en la Tabla 2, casi la quinta parte de los eyaculados tenían volúmenes inferiores a los $2.0 \mathrm{~mL}$ establecidos por la OMS como valor de referencia para los estudios de fertilidad; es más, un 6,3\% ni tan siquiera llegaban a los $0.5 \mathrm{~mL}$. Dado que eran varones de probada fertilidad y considerando que, tras la vasectomía, la pequeña reducción en el volumen es del orden de un 10 a un 15\%, existen más que fundadas sospechas de que los eyaculados no sean completos en bastantes más especímenes de los reconocidos, con la repercusión que esto pudiera tener en los resultados. En ningún artículo hemos encontrado una alusión al volumen de semen recogido; como mucho, se indica que se siguen las recomendaciones de la OMS con respecto al estudio seminal y se pasa directamente a analizar los recuentos observados.

Del mismo modo, en un 30,5\% de las muestras de semen se encontraron alteraciones de la consistencia, tanto de viscosidad como de licuefacción. De nuevo, en ningún artículo se analiza la repercusión que esto pudiera tener en la homogeneización del semen y en la consiguiente visualización de los espermatozoides, a pesar de las dificultades que tenemos los profesionales del laboratorio con el manejo de este tipo de muestras. Sin embargo, es obvio que, para obtener un resultado fiable, el semen debe estar muy bien homogeneizado, más aún cuando sabemos que es frecuente encontrar espermatozoides incluidos en fibras de moco o en grumos macro y microscópicos.

Otro aspecto analítico tampoco considerado en la literatura médica es la presencia de abundantes leucocitos $\mathrm{u}$ otras células en el examen directo y, sobre todo, en el sedimento; en nuestro caso no se pudo informar un resultado negativo en el 4,3\% de nuestros pacientes, a pesar de que no se observasen espermatozoides. Es importante reseñar que sólo 8 pacientes de este grupo habían venido después de las 16 semanas recomendadas y se sabe que al realizar el espermiograma en un momento demasiado cercano a la cirugía se puede encontrar una mayor respuesta celular6

Es evidente que las diferentes técnicas de análisis seminal empleadas por los distintos laboratorios pueden afectar los resultados y las decisio- nes clinicas subsecuentes. Evidentemente, para informar un resultado de azoospermia sin ningún margen de error deberíamos disponer de un método lo suficientemente fiable para no pasar por alto ningún espermatozoide $\mathrm{y}$, por supuesto, analizar todo el eyaculado. Sin embargo, los métodos disponibles en la actualidad distan mucho de ser ideales y, además, varían ampliamente; es curioso consultar en la literatura que unos laboratorios analizan semen en un porta; otros, en una cámara de recuento y otros, en una extensión de semen teñida. Otro punto nada desdeñable es el área visual analizada por cada laboratorio, que puede variar desde 20-30 campos a 400 aumentos en el examen directo hasta 300 campos de sedimento tras la centrifugación ${ }^{2,7,8}$. Cuanto más exhaustivo sea el análisis, lógicamente, habrá mayor probabilidad de observar espermatozoides y si, además, cuando se observan espermatozoides inmóviles se realiza la prueba de vitalidad, se aumenta la probabilidad de que el enfermo no consiga el alta. Es indudable que, en la mayoría de los casos, el ser demasiado exhaustivo no va a suponer ninguna ventaja práctica para el paciente, ya que estas diferencias, en realidad, no van probablemente a modificar el riesgo de gestación. A pesar de que como profesionales del laboratorio debemos tratar de ser lo más rigurosos posible en nuestros análisis, no debemos olvidar que también es importante colaborar con los clínicos en la interpretación de nuestros informes que, a fin de cuentas, son los que deben utilizar los resultados que emitimos.

Por otro lado, como para el resto de las magnitudes del laboratorio, sería mucho más correcto expresar la no visualización de espermatozoides como un recuento inferior al limite de detección de nuestra técnica y, así, se evitaría la confusión creada a los clínicos con términos como "reaparición de espermatozoides" tras la azoospermia o "posible recanalización" cuando, en realidad, nos movemos en un recuento extremadamente bajo de espermatozoides, que parecen “aparecer y desaparecer”. Por otro lado y, con el fin de unificar criterios entre todos los laboratorios, como en la literatura se considera el valor inferior a 10000 espermatozoides/mL como razonablemente aceptable ${ }^{3,9}$, recomendamos expresar un resultado negativo como inferior a 10000 
espermatozoides $/ \mathrm{mL}$, siempre que en el semen fresco no se observen espermatozoides o bien el recuento de los espermatozoides muertos/inmóviles sea lo suficientemente pequeño como para que éste no se pueda realizar en cámara de Neubauer. Por supuesto, el laboratorio debe seguir centrifugando el semen para comprobar que no existen espermatozoides vivos aún en bajísimas concentraciones y realizando tinciones de vitalidad, si las condiciones preanalíticas lo permiten, para comprobar que no existen espermatozoides vivos.

Por último, al igual que otros autores ${ }^{10}$, nos habíamos cuestionado la necesidad de realizar, al menos, dos espermiogramas por paciente. A pesar de las recomendaciones, con las implicaciones legales que pudieran existir en caso de embarazo, muchos pacientes se conforman con un único resultado satisfactorio. Lógicamente, un paciente que ha obtenido un primer informe de azoospermia es mucho más difícil que colabore trayendo un nuevo eyaculado que uno al que se le han encontrado espermatozoides. Sin embargo, dejando de lado los problemas legales $\mathrm{y}$, a la vista de nuestros resultados, sólo la frecuente incidencia de problemas a lo largo del proceso analítico justifica ampliamente la necesidad de realizar un segundo análisis.

\section{CONCLUSIONES}

En conclusión, como se deduce de nuestros resultados, la incidencia de problemas preanalíti$\cos \mathrm{y}$ analíticos podría alterar los resultados en casi un tercio de los espermiogramas evaluados y explicar gran parte de las aparentes "apariciones y desapariciones" de espermatozoides, justificando el examen de un mínimo de dos especímenes de semen por paciente. Además, las diferentes técnicas de estudio seminal pueden ser responsables de amplias variaciones en los resultados, que también podrían explicar las fluctuaciones en la visualización o no de los espermatozoides.
Por último y no menos importante, la correcta expresión de los resultados de este tipo de espermiogramas como un recuento inferior al límite de detección de nuestra técnica, al igual que se viene realizando para otras magnitudes, solucionaría por sí sola gran parte de los problemas derivados de la estandarización del proceso postanalítico.

\section{REFERENCIAS}

1. Smith JC, Cranston D, O'Brien T, Guillebaud J, Hindmarsh J, Turner AG. Fatherhood without apparent spermatozoa after vasectomy. Lancet. 1994 Jul 2;344 (8914):30.

2. Knijff DW, Vrijhof HJ, Arends J, Janknegt RA. Persistence or reappearance of nonmotile sperm after vasectomy: does it have clinical consequences? Fertil Steril. 1997;67(2): 332-335.

3. O`Brien TS, Cranston D, Ashwin P, Turner E, MacKenzie IZ, Guillebaud J. Temporary reappearance of sperm 12 months after vasectomy clearance. Br J Urol. 1995;76(3): 371-372.

4. Cháfer M, Navarro L, Fuster O, Andrés C, Vera J, Domínguez JP. Protocolo para el control de la vasectomía. Diag Biol. 2006;55(1):55-59.

5. Hancock P, McLaughlin E. British Andrology Society guidelines for the assessment of post vasectomy semen samples (2002). J Clin Pathol. 2002;55(11):812-816.

6. World Health Organization. Laboratory manual for the examination of human semen and sperm cervical mucus interaction, 4th ed. Cambridge; Cambridge University Press, UK, 1999.

7. Edwards IS. Earlier testing after vasectomy, based on the absence of motile sperm. Fertil Steril. 1993;59(2):431-436.

8. Bradshaw HD, Rosario DJ, James MJ, Boucher NR. Review o current practice to establish success after vasectomy. Br J Surg. 2001;88(2):290-293.

9. Davies AH, Sharp RJ, Cranston D, Mitchell RG. The longterm outcome following "special clearance" after vasectomy. BR J Urol. 1990;66(2):211-212.

10. Badrakumar C, Gogoi NK, Sundaram SK. Semen analysis after vasectomy: when and how many? BJU Int. 2000; 86(4):479-481.

Correspondencia autor: Dra. M. Cháfer Rudilla

Servicio de Análisis Clínicos. Compejo Hospitalario y Universitario de Albacete. Hermanos Falcó, 37. 02006 Albacete.

Tel.: 967597100

Información artículo: Original - Esterilización

E-mail autor: mcharud@telefonica.net

Trabajo recibido: septiembre 2006

Trabajo aceptado: febrero 2007 